\title{
Former Food Products Safety Evaluation: Computer Vision as an Innovative Approach for the Packaging Remnants Detection
}

\author{
Marco Tretola, ${ }^{1}$ Matteo Ottoboni, ${ }^{1}$ Ambra Rita Di Rosa, ${ }^{2}$ \\ Carlotta Giromini, ${ }^{1}$ Eleonora Fusi, ${ }^{1}$ Raffaella Rebucci, ${ }^{1}$ Francesco Leone, ${ }^{2}$ \\ Vittorio Dell'Orto, ${ }^{1}$ Vincenzo Chiofalo, ${ }^{2}$ and Luciano Pinotti ${ }^{1}$ \\ ${ }^{1}$ Department of Health, Animal Science and Food Safety, Università degli Studi di Milano, 20134 Milano, Italy \\ ${ }^{2}$ Department of Veterinary Science, Università degli Studi di Messina, 98168 Messina, Italy
}

Correspondence should be addressed to Carlotta Giromini; carlotta.giromini@unimi.it

Received 24 March 2017; Accepted 21 May 2017; Published 12 June 2017

Academic Editor: Valber A. Pedrosa

Copyright (c) 2017 Marco Tretola et al. This is an open access article distributed under the Creative Commons Attribution License, which permits unrestricted use, distribution, and reproduction in any medium, provided the original work is properly cited.

Former food products (FFPs) represent a way by which leftovers from the food industry (e.g., biscuits, bread, breakfast cereals, chocolate bars, pasta, savoury snacks, and sweets) are converted into ingredients for the feed industry, thereby keeping food losses in the food chain. FFPs represent an alternative source of nutrients for animal feeding. However, beyond their nutritional value, the use of FFPs in animal feeding implies also safety issues, such as those related to the presence of packaging remnants. These contaminants might reside in FFP during food processing (e.g., collection, unpacking, mixing, grinding, and drying). Nowadays, artificial senses are widely used for the detection of foreign material in food and all of them involve computer vision. Computer vision technique provides detailed pixel-based characterizations of colours spectrum of food products, suitable for quality evaluation. The application of computer vision for a rapid qualitative screening of FFP's safety features, in particular for the detection of packaging remnants, has been recently tested. This paper presents the basic principles, the advantages, and disadvantages of the computer vision method with an evaluation of its potential in the detection of packaging remnants in FFP.

\section{Introduction}

To meet the growing global demand for food of animal origin, the development of livestock production will increase rapidly at global level. Feed and food security is high on the agenda for government and retailers, so research into alternative feed ingredients for a competitive/efficient and cost effective animal production is important. This implies that the processes of innovation and development in animal nutrition should not be considered concluded. In this respect, the use of alternative raw materials in farm animal's diets, to improve sustainability, has been becoming also important. Concurrently, the effect of feed ingredients on growth performance of host animals should be carefully accounted for proper evaluation of an alternative ingredient [1]. Feed quality and issues surrounding the safety of raw materials are always of interest to all livestock farmers and feed manufacturers. These concerns are even more important when alternative feed ingredients, new product developments, and innovative feeding trends are considered. In this context, the case of former food products (FFPs) represents an exhaustive example [2]. According to the EU Catalogue of Feed Materials [3], "former foodstuffs are foodstuffs, other than catering reflux, which were manufactured for human consumption in full compliance with the EU food law but which are no longer intended for human consumption for practical or logistical reasons or due to problems of manufacturing or packaging defects or other defects and which do not present any health risks when used as feed." Thus, FFPs represent a way by which losses are converted from the food industry into ingredients for the feed industry, thereby keeping food losses in the food chain [4]. Of note, operations behind the FFPs production process have nothing to do with waste processing or food waste recycling.

Processing methods to convert former food into feed ingredients do not usually include preremoval of packaging 
TABLE 1: Nutrient composition of FFPs evaluated in two different studies, barley and wheat.

\begin{tabular}{lcccc}
\hline & FFPs [10] & FFPs [11] & Barley & Wheat \\
\hline Crude protein (\%) & 10.9 & 10 & 11 & 12.4 \\
Crude fat (\%) & 9.8 & 10.1 & 2.8 & 2.1 \\
Crude fibre (\%) & 2.2 & 2.7 & 5.5 & 2.7 \\
Starch (\%) & 41.9 & 52.4 & 51.6 & 59.2 \\
Sugar (\%) & 14 & 19.6 & 2.2 & 2.4 \\
Metabolizable energy (ME) for pigs (MJ/kg) & 15.48 & 16.9 & 12.95 \\
\hline
\end{tabular}

materials. Consequently, although feed processors routinely remove the packaging from ex-food mechanically in the feed plant, the final product appears to be irremediably contaminated by small amounts of wrapping materials [5]. In order to evaluate any "accepted" level of risk, Raamsdonk and coworkers (2012) [6] validated a method for detection and quantification of packaging materials in FFPs that are intended to be used as feed material. This method is based on the separation of each nonnative particle from the FFPs matrix by bare eye examination to calculate the percentage (w/w) of the amount of packing remnants. Nevertheless, validated method can be considered very laborious, time consuming, and subjective. In light of this, an innovative approach, based on computer vision coupled with stereomicroscopy, has been recently considered for a more rapid food packaging remnants detection [7].

The computer vision (CV) technique has been proved to be a rapid qualitative screening approach to estimate the presence of foreign materials in food and feed allowing the reduction of tedious and subjective human visual involvement $[2,7-9]$.

This paper is focused on the description of basic principles of the two methods and their application in food and feed quality assessment. In particular, the strengths and limits of both techniques/approaches in the detection of packaging remnants in FFPs are also discussed.

\section{Former Foodstuff Products and Packaging Remnant Contamination}

FFPs represent an opportunity to convert food losses into feed ingredients, avoiding the waste of resources from the food chain, in line with the circular-economy concept. As previously reported $[13,14]$, a balanced combination of byproducts can be considered an opportunity to substitute the traditional energy and protein sources in farm animals' nutrition without major changes in the diet composition, with positive environmental and economic returns. About 3,5 million tonnes of former foodstuffs are processed into feed in the EU [15]. In a recent study, the nutritional values of FFPs were evaluated [11]. According to their original composition, FFPs can maintain a high nutritional potential in terms of nutrients content and energy values for feeding animals, representing a further category of alternative feed ingredients. Moreover, Giromini et al. [11] observed that the FFPs nutrient composition is comparable to that of cereal grains but with higher fat content. Furthermore, the relatively high NSC/starch ratio and fat concentrations design FFPs as valuable energetic feed ingredients with notable digestible and metabolizable energy content. These nutritional features are in line with other processed former foodstuffs [10] (Table 1). Moreover, FFPs showed in vitro digestibility values comparable to those obtained for wheat [11]. On the basis of these findings, FFPs can be considered an energy-fortified version of common cereals grains with promising application as nontraditional ingredients for animal feed, in particular for swine nutrition. Despite the nutritional quality of FFPs, on the other hand, they may represent a challenge regarding safety aspects. Depending on the type of ex-food, their revalorisation in the feed sector implies a combination of different processes such as collection, unpacking, mixing, grinding, and drying, which might affect not only quality but also their safety. Exfood, though nutritious and stable from a microbiological point of view, might generate other safety issues, such as those related to the presence of packaging remnants. One of the main categories of former food products intended for feeding purposes is bakery co-by-products such as bread, biscuits, waffles, and breakfast cereals. In the current practice of food production, proper packaging materials are essential for assuring quality maintenance during transport and storage. Materials used for food packaging can vary widely and their original appearance can vary a lot based on material characteristics and on the processing method adopted during the feed production process. Paper, board, and carton, for example, change their original appearance producing paper fibres, with high abundance in the FFPs [16]. The most common packaging material in food industries is plastic, even though resin, aluminium, and pressed paperboard are also widely used. Specifically, the main types of material used in food packaging are polyolefin such as polypropylene (usually made in black or clear, very rigid, crack-resistant; it can resist temperature up to 220 to $240^{\circ} \mathrm{C}$ ) and polyethylene (with an average melting point of $120^{\circ} \mathrm{C}$ [17]); polyethylene terephthalate and its copolymers (PET that can be coloured and starts to melt before $140^{\circ} \mathrm{C}$ ); polystyrene (which can be white, black, green, pink, yellow, or blue with a moderate temperature resistance); rigid polyvinylchloride (PVC, regenerated cellulose $(\mathrm{RC})$ ), pressed paperboard (made in a variety of colours and patterns and can be placed in the oven up to $200^{\circ} \mathrm{C}$ for one hour); and aluminium foil (silver or coated in colours and can withstand very high heat) [18]. In the feed processing chain, about $80 \%$ of the biscuit products 
are processes unpacked, while the bread is predominantly processes packaged. FFPs are also composed by other dry products including sweets and dairy powders. Finally, FFPs in few cases include also wet products (i.e., beverages) [16]. Despite removal of packaging by feed processors, packaging materials can be found in the final product [5]. Specifically, plastics, paper, and aluminium foils are the most common packaging remnants in former food [19].

European Union Member States generally agree that a zero tolerance for these traces is neither practical nor proportionate to the risk and not all authorities agree on this point. The Netherlands and German authorities have undertaken their own risk assessments and both countries now tolerate the presence of packaging up to a level of 0.15\% [16]. The UK Food Standard Agency had adopted a tolerance of $0.15 \%$ for such residues, while no limits are assessed in European legislation [16]. In this scenario, the need of appropriate methods for establishing the level of contamination with remnants of packaging materials in FFPs intended for animal feeding is crucial.

\section{Detection of Packaging Materials in FFP: A Comparison of Existing and Innovative Methods}

Recently, at the RIKILT Institute, Raamsdonk and coworkers (2012) [6] validated a method for detection and quantification of packaging materials in mesh bakery products. The validated method for bakery products can be summarised as (1) visual selection of undesired ingredients which can be identified as remnants of packaging materials, (2) weighing of the selected materials, (3) defatting and dehydration (if necessary), (4) final weighing, (5) reporting of weight and percentage. The basic principle is to select and separate every particle that is not native to the matrix by bare eye examination. In addition, a similar method for pelleted samples has been recently validated by Marchis and coauthors [5].

In the Dutch monitoring program for feed materials (2005-2010), a total of 160 samples of bakery products have been collected and analyzed [16]. Based on the tolerance level of $0.15 \%$ determined by Netherlands and German authorities, more than $90 \%$ of all samples investigated showed a level of presumed packaging remnants below this threshold and the annual average was between $0.03 \% \mathrm{w} / \mathrm{w}$ and $0.06 \% \mathrm{w} / \mathrm{w}$ [16].

In a recent study [12], six samples of mixed FFPs, mainly composed of bakery products collected in Italy, have been analyzed using the validated method [6]. In addition, in this study, the packaging remnant contamination level in pelleted FFPs samples has been also evaluated, combining the conventional method to the wet sieving procedure according to Marchis and coauthors (2016) [5] and Miladinovic (2009) [20]. The results obtained were in line with those reported by Raamsdonk and coworkers [6]: the packaging remnants contamination level in the bakery products collected in Italy was below the threshold level indicated by both Netherlands and German authorities. Moreover, the packaging remnants were more frequently found in the 1-millimeter sieve mesh fractions (Figure 1) except for two samples (Figure 1) probably due to the low level of contaminants and to the row

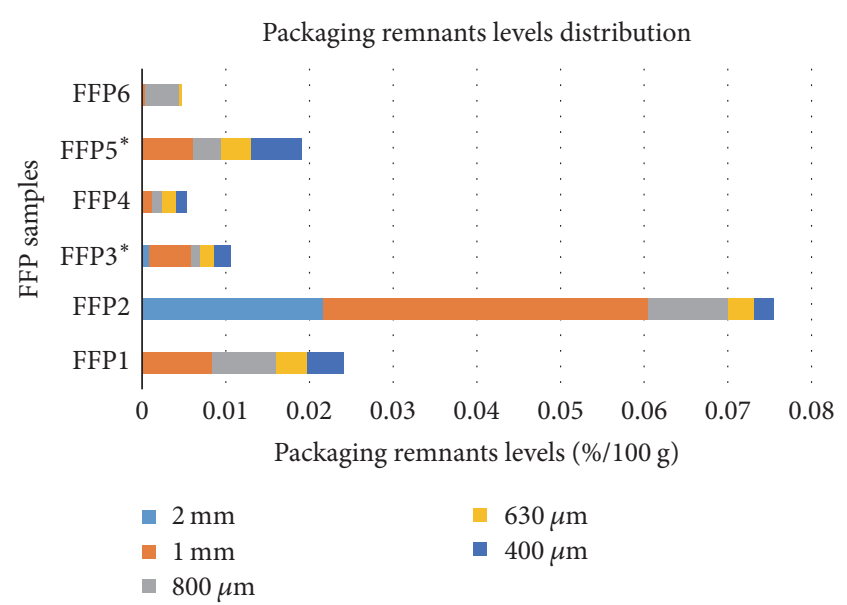

FIGURE 1: Packaging remnants contamination (\% $\mathrm{w} / \mathrm{w})$ in all FFPs samples, divided for different sieve mash fractions. ${ }^{*}$ Pelleted samples (adapted from [12]).

ingredients of the samples. Packaging remnants particle size and nature may affect remnants segregation and distribution in the different fractions. Raamsdonk et al. (2012) [6] demonstrated that the difficulty of recognizing and picking up of particles largely depends on their size. Particles smaller than $1 \mathrm{~mm}$ are very difficult to handle and it has been suggested [6] that it is not necessary to separate the fraction with the smaller particles $(<500 \mu \mathrm{m})$. Likewise, recognition and quantification of particles smaller than the sieve mesh size of $400 \mu \mathrm{m}$ are difficult; therefore, these particles may be excluded from the total quantification [12]. The validated method has proven to be effective in the quantification of packaging materials also in low contaminated FFP samples; it is suitable in the evaluation of the heterogeneous remnants distribution among different sieve mash fractions both in mesh and in pelleted samples [5] and it can be useful in the recognition of the remnants nature, although with some exceptions. However, this method is laborious and operatordependent and the amount of contaminants could be underestimated in a sample due to the inability of collecting and quantifying remnants from the smallest sieve mashes.

Nowadays, different techniques are used for the detection of foreign materials in food, usually combined with an automated processing and removal system [21-23] and all of them involve computer vision (CV). CV can be defined as an automated process that makes use of a computer to classify data extracted from an object in the form of image [24]. The number of available technology based on imaging hardware and decision algorithms is growing in order to decrease the operator inspection. For this purpose, CV methods are being used increasingly in the food industry for quality assurance. In particular, CV is designed for advanced colour analysis of complex surfaces of food, beverage, and packaging products and, thanks to the advanced multivariate statistics processing (i.e., Statistical Quality Control), is able to perform in-depth analysis of complex images. CV can be considered a promising technique currently developed for food colour measurement, especially for its ability in 
providing a detailed characterization of colour uniformity at pixel-based level [8]. In this context, the CV System (IRIS Visual Analyzer VA400) has been considered for its ability in the characterization of foreign materials in several food matrices. The CV analysis starts positioning sample on the available surface and submits them to top and bottom controlled white lighting conditions $\left(6500^{\circ} \mathrm{K}\right)$ to avoid shadow effects. In this regard, the nature of the illuminants and their position are considered essential factors to contemplate in order to improve the accuracy and reduce the time and complexity of the subsequent image processing analysis [25]. The most used illuminants are fluorescent and incandescent bulbs, even though also light-emitting diodes (LEDs) and electroluminescent sources are used [8]. For samples with flat surface, the ring illuminator is commonly used while the diffuse illuminator is suitable for products with sphere shape, providing a diffuse illumination on the sample surface [8]. Despite the importance of the illumination, the core of $\mathrm{CV}$ is the image processing and analysis [26]. Pictures of sample surface are taken by the use of a high resolution CCD camera (16 million colours). The subsequent image processing/analysis can be divided in a low level processing, which includes image preprocessing, improving the image quality by suppressing undesired distortions; intermediate level processing involves image segmentation (division of the image into regions strongly correlated with areas of interest), representation (evaluation of image texture and defects), and description (extraction of quantitative information from the previously segmented image regions); high level processing which uses statistical analysis and neural networks to obtain information about food quality, texture, and grading [27]. CV System captures the colour of each pixel within the image using three colour sensors per pixel [28], specifically capturing the intensity of the light in the red, green, and blue spectrum in the RGB (red, green, and blue) space. It is the most popular hardware-oriented colour space through which cameras sense the environment [29] and considered one of the best approaches to evaluate also a very small amount of colour variation [30]. Usually, through image processing and analysis, CV obtains colour information from pixels of digital image acquired by a high resolution CCD (chargecoupled device) camera, obtaining 16-million colours images, but it is also able to perform analyzes on images from external devices. For each picture, the colour spectrum of the sample is represented in the form of histogram that can be considered a colour map of the object [31]. The proportion of each colour is represented by the software as a percentage. Starting from the colour spectrum obtained from sample pictures, the software of the CV System can be used to build up the Statistical Quality Control (SQC) chart, constructed with two areas (conforming and nonconforming) based on statistical test which helps the user to make a decision with a certain error probability [31]. In this qualitative application, in order to obtain a model relating the sensor data to a quality, a training phase to relate the variability of the product to the sensor data recorded by the analysis system is needed. This step is necessary to obtain the SQC chart where conforming and nonconforming areas are represented. Based on responses of each sensor, unknown samples analyzed are mapped onto

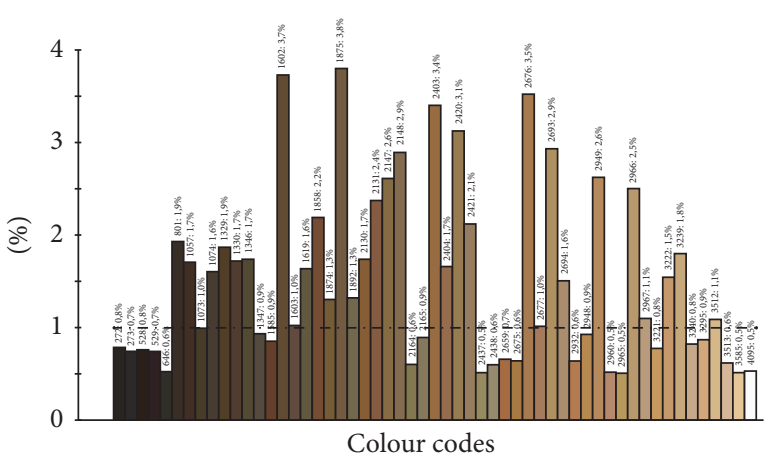

FIGURE 2: Example of the histogram representation of the colour spectrum of a single FFP's picture obtained by the use of digital camera (CoolSNAP-Pro colour camera, 6.3x) and analyzed by CVS AlphaSoft software (Alpha MOS, France). On a fixed scale of 4096 colours, the proportion of each colour in the analyzed image is represented as a percentage.

the chart in the corresponding area [31]. In this statistical approach, it is necessary to calculate average value $(\mathrm{mj})$ and a standard deviation $(\delta j)$ from the responses of each sensor " $j$." The software expresses the difference between conforming training samples and unknown sample to be checked as a distance [31]. To set tolerance area of the SQC graph, the software uses the following acceptability index (or rejection probability): $I_{\text {acceptability }}=\left(1-\mathrm{P}_{\text {rejection }}\right) \times 100$ with $0<\mathrm{P}_{\text {rejection }}<1$. In this scenario, Tretola and coauthors [12] have compared the validated method with the CV coupled with stereomicroscopy, as an innovative, rapid, and qualitative (presence/absence) approach for the evaluation of FFPs packaging contamination. Due to the small size of samples particles, it was not possible to obtain high resolution pictures for an appropriate image analysis by the use of a high resolution CV System CCD camera (IRIS Visual Analyzer VA400). For this reason, the CVS technique was coupled with stereomicroscope image analysis. Each sample was observed using a stereomicroscope (Olympus SZX9; Tokyo, Japan). By the use of a digital camera (CoolSNAP-Pro colour camera), pictures $(6.3 \mathrm{x})$ obtained by stereomicroscope were processed as a colour spectrum in the RGB space across the surface of FFP samples using CVS AlphaSoft software (Alpha MOS, France). An example of the histogram representation of the colour spectrum obtained by the analyses of a contaminated FFP sample's picture is showed in Figure 2. As shown in the histogram, the colours of FFPs can vary a lot, especially in pictures containing coloured food residues (coming from the native raw material). Indeed, the contaminants in FFPs can include a wide range of colours, making it very difficult to distinguish packaging remnants from the background feed colours. In spite of that, Tretola and coauthors [12] found a discriminant colour code (4095), which always occurs in FFP's pictures where the presence of packaging remnants can be observed. The 4095 seems to be associated with the reflecting properties of packaging remnants. An example of the percentage of this colour code 4095 detected by the CV in a picture of a contaminated FFP sample is indicated in the last column of Figure 2. Afterwards, the SQC model was 
TABLE 2: Advantages and drawbacks of existing and innovative methods.

\begin{tabular}{lll}
\hline Method & Advantages & Drawbacks \\
\hline \multirow{2}{*}{ Existing* $^{*}$} & $\begin{array}{l}\text { (i) Quantification of major packaging remnants } \\
\text { (ii) Evaluation of the heterogeneous remnants distribution } \\
\text { (iii) Determination of packaging remnants nature }\end{array}$ & $\begin{array}{l}\text { (i) Limited quantification for small packaging remnants } \\
\text { (ii) Laborious/time consuming } \\
\text { (iii) Operator-dependent }\end{array}$ \\
\hline & $\begin{array}{l}\text { (i) Rapidity } \\
\text { (ii) Objectivity } \\
\text { Innovative** }\end{array}$ & $\begin{array}{l}\text { (i) No quantification } \\
\text { (ii) No determination of packaging remnants nature } \\
\text { (iv) Remote sample image analysis }\end{array}$ \\
\hline
\end{tabular}

${ }^{*}$ From [6]. ${ }^{* *}$ From [12].

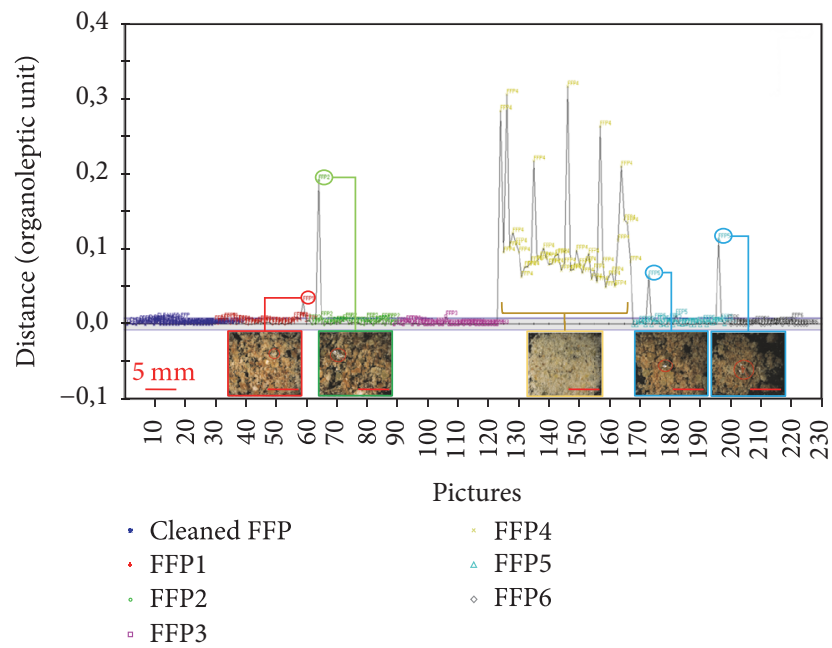

FIGURE 3: SQC chart (adapted from [12]). Each symbol represents a picture of the corresponding sample, based on its colour. The grey area indicates the range distance obtained by the use of cleaned FFP sample in the training phase. Symbols plotted outside the grey band indicate sample's pictures characterized by the presence of packaging remnants, specifically significant percentage of the colour code 4095.

used to automatically perform the visual quality control of FFPs. Based on the presence in the FFPs colour spectrum of the discriminant colour code, the study demonstrated the ability of CVS to detect the presence of packaging remnants in pictures of contaminated FFPs samples. Specifically, in the SQC chart where a cleaned FFP was used to determine the area of tolerance, all pictures showing the presence of packaging remnants were plotted outside this threshold, indicating a significant distance from the cleaned FFP due to the presence of foreign materials (Figure 3). As shown in Figure 3, the distance value of each picture is given by the total percentage of colour code 4095. Increasing the packaging remnants size in the picture (specifically the light reflecting part) increases the distance from the cleaned FFPs sample. The most distant sample from the cleaned sample contains a lot of light spots in all pictures; the distance is given by the sum of these spots in each picture. Pictures within the threshold value do not contain packaging remnants, with no significant 4095 colour code percentage and for this reason they are considered close to the cleaned FFP by the SQC. In the same study, the ability of CVS to detect very small presumed packaging particles has been also tested. For instance, the FFP4 sample was plotted as nonconforming by CVS analysis (Figure 3), whereas it was classified among the cleanest samples (Figure 1), using the validated method; all pictures were plotted as nonconforming by the cleaned FFP sample in the CVS analysis. The nature of these presumed packaging materials, however, was not determined. In light of this, the impossibility to obtain pictures directly by the CV System and the consequent lack of a homogeneous illumination during the image acquisition, by the use of stereomicroscope, can be considered a weakness that decreases the accuracy of the subsequent image processing steps. In fact, illumination is a key issue. As reported elsewhere [8], illumination can greatly affect the quality of captured image. Different illuminants may yield different stimuli using the same camera. A well-designed illumination system can improve the accuracy, reduce the time and complexity of the subsequent image processing steps, lead to success of image analysis, and decrease the cost of an image processing system. This is particularly true for material like FFP. Due to the irregular particle size and shape of FFPs, they can form shadows very difficult to eliminate by image processing technique. On the other hand, the CV coupled with stereomicroscopy approach demonstrated that excellent potential to adapt this technique in the safety evaluation of former foodstuffs exists, but improvement of the system's CCD camera in order to obtain high resolution pictures, in a uniform illumination environment, is a crucial step to adequate detection identification.

\section{Conclusion}

Food quality research requires an improvement of the available methods for foodstuff inspection. In particular, the current ex-food processing techniques in the feed industry cannot completely remove packing remnants, affecting the safety of FFPs. In light of that, appropriate methods to evaluate the contamination with remnants of packaging materials in FFPs represent a current field of research. The validated method has proven to be effective in monitoring the contamination levels with a tolerance limit higher than zero in FFPs. Moreover, it is suitable in the evaluation of the heterogeneous remnants distribution among different sieve mashes fraction both in mesh and in pelleted samples. However, this method is very laborious and operator-dependent. It also presents limitations related to the inability of detecting remnants from the smallest sieve mashes. Conversely, (Table 2) the 
innovative method based on a CV System coupled with stereomicroscope has shown a potential in detecting efficiently, rapidly, and more objectively the presence/absence of presumed packaging remnants also in low contaminated ex-food. CV System coupled with stereomicroscope has the potential of becoming a promising tool for FFPs safety evaluation. This method could integrate the validated method for a rapid qualitative evaluation of FFPs safety. However, the implementation of the CCD camera magnification seems to be a prerequisite to refine and to facilitate the application of CV coupled with stereomicroscope in FFPs safety evaluation. In fact, increased CV capabilities along with its flexibility and nondestructive nature could make this method suitable for the FFP safety evaluation in an automated feed processing chain.

\section{Conflicts of Interest}

The authors declare that there are no conflicts of interest regarding the publication of this manuscript.

\section{References}

[1] R. Swick, "Considerations in using protein meal for poultry and swine," ASA technical Bulletin, vol. AN, 1999.

[2] L. Pinotti, C. Giromini, M. Ottoboni, M. Tretola, F. Cheli, and A. Baldi, "Ex-food 4 feed: quality and safety," in Celebrating Food: Proceedings, University of Novi Sad, Novi Sad, Serbia, 1st edition, 2016.

[3] European Commission, "Commission Regulation (EC) No 68/2013 of the European Parliament and of the Council, on the catalogue of feed materials," Official Journal of the European Union, no. 1, 2013, L29.

[4] P. Featherstone, Challenges in Global Raw Material Supply: New Raw Materials, 2016.

[5] D. Marchis, T. Giovannini, G. Amato, L. Ceppa, L. Pinotti, and M. Gili, "Development and validation of a method for detection and quantification of packaging residues in feed from former food," in Proceedings of the 5th International Feed, Book of Abstract FEED '16, p. 59, Geel, Belgium, October 2016.

[6] L. V. Raamsdonk, V. Pinckaers, J. Vliege, and H. V. Egmond, Examination of Packaging Materials in Bakery Products: A Validated Method for Detection and Quantification RIKILT Report 2012.007, 2012.

[7] L. Pinotti, "Image analysis and microscopy: a useful combination," Biotechnologie, Agronomie, Société et Environnement, vol. 13, pp. 21-24, 2009.

[8] D. Wu and D.-W. Sun, "Colour measurements by computer vision for food quality control-a review," Trends in Food Science \& Technology, vol. 29, no. 1, pp. 5-20, 2013.

[9] L. Pinotti, T. Fearn, S. Gulalp et al., "Computer image analysis: an additional tool for the identification of processed poultry and mammal protein containing bones," Food Additives and Contaminants-Part A Chemistry, Analysis, Control, Exposure and Risk Assessment, vol. 30, no. 10, pp. 1745-1751, 2013.

[10] A. Bouxin, "Feed Use of Former Foodstuffs: Converting Food into Food," FEFAC, 2012.

[11] C. Giromini, M. Ottoboni, M. Tretola et al., "Nutritional evaluation of former food products (ex-food) intended for pig nutrition," Food Additives \& Contaminants: Part A, pp. 1-10, 2017.
[12] M. Tretola, A. R. Di Rosa, E. Tirloni et al., "Former food products safety: microbiological quality and computer vision evaluation of packaging remnants contamination," Food Additives \& Contaminants: Part A, 2017.

[13] L. Pinotti, A. Krogdahl, I. Givens et al., "The role of animal nutrition in designing optimal foods of animal origin as reviewed by the COST action feed for health (FA0802)," Biotechnology, Agronomy and Society and Environment, no. 4, pp. 18-47, 2014.

[14] L. Pinotti and V. Dell'Orto, "Feed safety in the feed supply chain," Biotechnology, Agronomy and Society and Environment, vol. 15, no. 1, pp. 9-14, 2011.

[15] G. Lannoy, "Keeping food losses in the food chain," in Proceedings of the OECD Food Waste in Retail and Processing Conference, 2016.

[16] L. V. Raamsdonk, R. Rijk, G. Schouten et al., A Risk Evaluation of Traces of Packaging Materials in Former Food Products Intended as Feed Materials RIKILT Report 2011.002, 2011.

[17] J. Ratzlaff, Polyethylene: Process Sensitivity in Rotational Molding, Chevron Phillips Chemical Company LP, Texas, Tex, USA, 2004.

[18] “Types of Material Used in Food Packaging," oak packaging, http://www.oakpackaging.com/include/assets/Materials.pdf.

[19] Regulation (EC) No 1935/2004 of the European Parliament and of the Council on materials and articles intended to come into contact with food and repealing Directives 80/590/EEC and 89/109/EEC, 2004.

[20] D. Miladinovic, Wet Sieving Procedure on a 'Retsch AS 200 Control' for Feed Manufactured Pellets or Feed Compound, Fôrtek, Norwegian University of Life Science, Ås, Norway, 2009.

[21] M. Ruiz-Altisent, L. Ruiz-Garcia, G. P. Moreda et al., "Sensors for product characterization and quality of specialty crops-a review," Computers and Electronics in Agriculture, vol. 74, no. 2, pp. 176-194, 2010.

[22] S. N. Jha and T. Matsuoka, "Non-Destructive Techniques for Quality Evaluation of Intact Fruits and Vegetables," Food Science and Technology Research, vol. 6, no. 4, pp. 248-251, 2000.

[23] R. P. Haff and N. Toyofuku, "X-ray detection of defects and contaminants in the food industry," Sensing and Instrumentation for Food Quality and Safety, vol. 2, no. 4, pp. 262-273, 2008.

[24] D. Sun, Computer Vision Technology in The Food and Beverage Industries, Elsevier, London, UK, 2012.

[25] C. Du and D. Sun, "Recent developments in the applications of image processing techniques for food quality evaluation," Trends in Food Science Technology, vol. 15, pp. 230-249, 2004.

[26] G. Krutz, H. Gibson, D. L. Cassens, and M. Zhang, "Colour vision in forest and wood engineering," Landwards, vol. 55, pp. 2-9, 2000.

[27] T. Brosnan and D. W. Sun, "Improving quality inspection of food products by computer vision-a review," Journal of Food Engineering, vol. 61, no. 1, pp. 3-16, 2004.

[28] D. Forsyth and J. Ponce, Computer Vision: A Modern Approach, Prentice Hall, New Jersey, NJ, USA, 2003.

[29] J. Russ, The Image Processing Handbook, CRC Press, Boca Raton, Fla, USA, 5th edition, 1999.

[30] M. M. Lana, L. M. M. Tijskens, and O. Van Kooten, "Effects of storage temperature and fruit ripening on firmness of fresh cut tomatoes," Postharvest Biology and Technology, vol. 35, no. 1, pp. 87-95, 2005.

[31] M. O. S. Alpha, "IRIS Visual AnalyzerAlpha Soft V14 Manual," http://www.alpha-mos.com. 

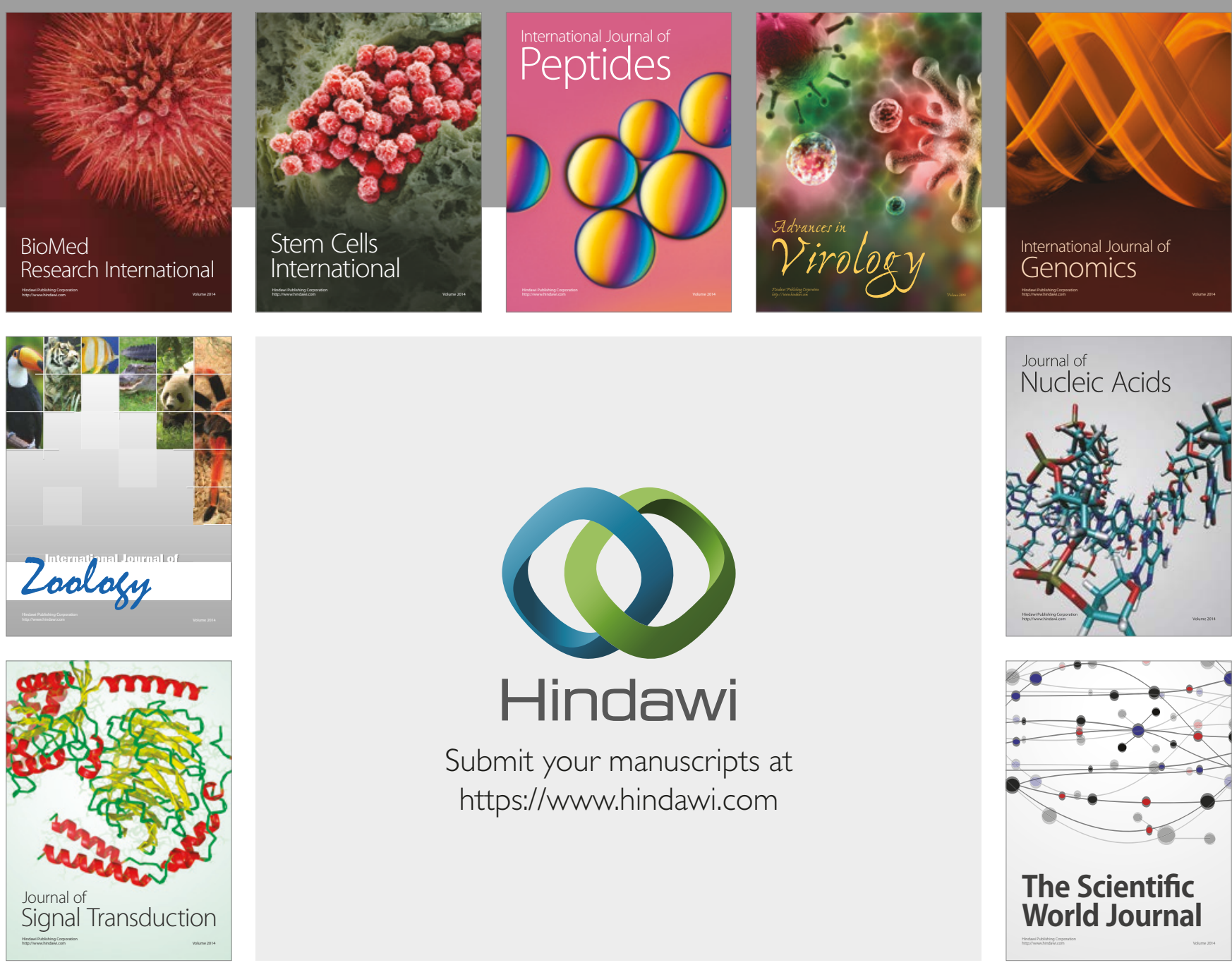

Submit your manuscripts at

https://www.hindawi.com
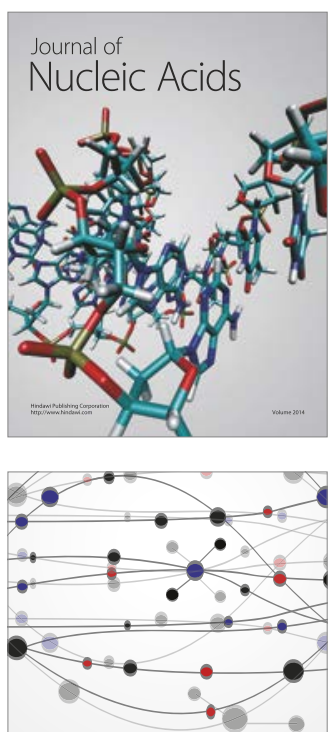

The Scientific World Journal

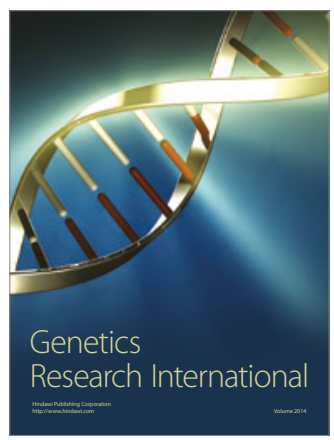

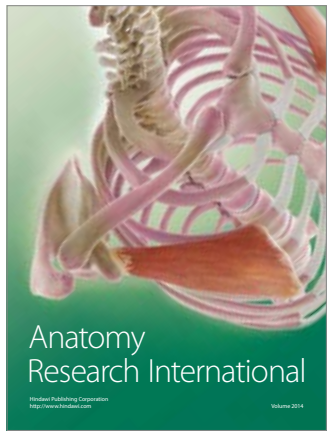

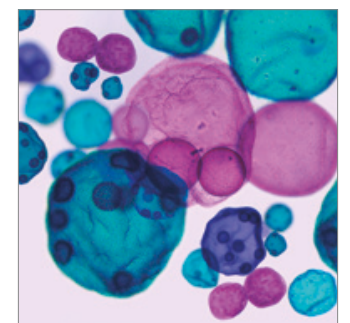

International Journal of Microbiology
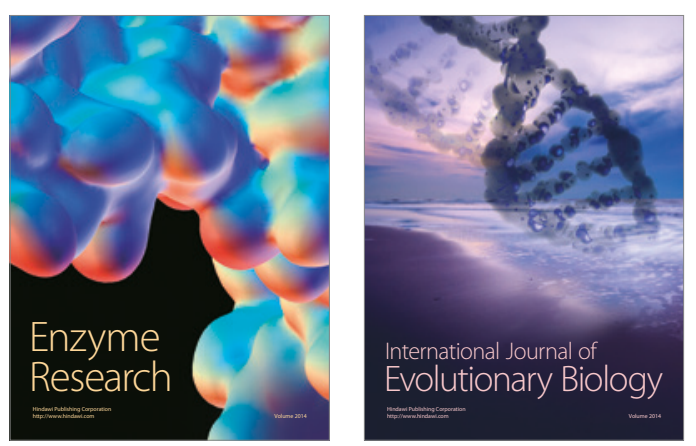
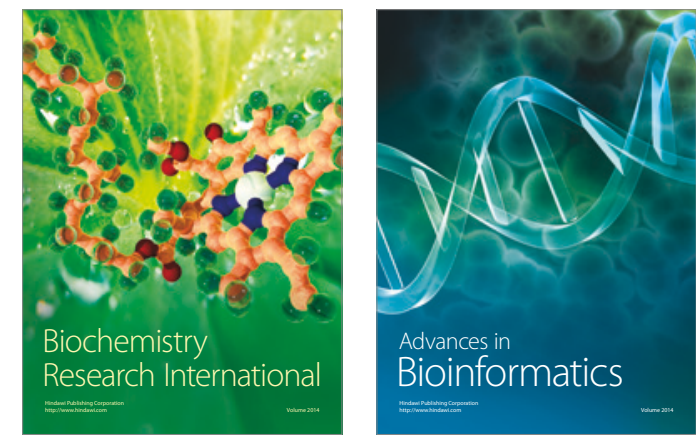

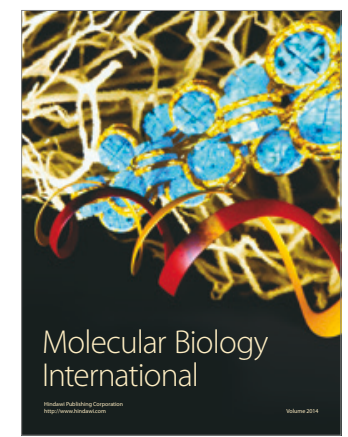

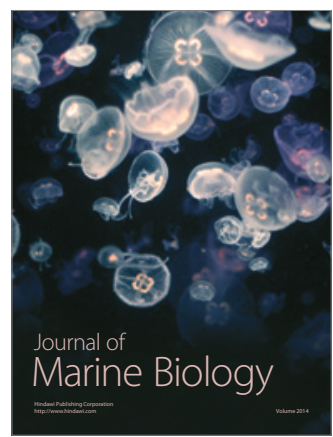

